\title{
AVALIAÇÃO DA COMPOSIÇÃO MINERAL DAS FOLHAS DE SENE POR ESPECTROMETRIA DE ABSORÇÃO ATÔMICA
}

\author{
Marina D. Dalaqua, Paula F. Brisolla, Cesar V. Nascimento, Marcos A. Zocoler \\ Universidade do Oeste Paulista - UNOESTE. Curso de Farmácia, Presidente Prudente - SP. E-mail: zocoler@unoeste.br
}

\begin{abstract}
RESUMO
Alguns nutrientes minerais presentes em plantas são considerados essenciais à saúde por atuarem em importantes vias metabólicas necessárias à vida humana. Porém, é importante ressaltar que níveis elevados desses minerais podem ser perigosos e tóxicos ao organismo. A cultura brasileira popularizou o consumo de sene, Senna alexandrina Miller, uma droga vegetal utilizada em dietas para emagrecimento. Nesse sentido, o objetivo deste trabalho foi avaliar as concentrações de metais pesados e os teores dos elementos minerais, por espectrometria de absorção atômica, presentes nas folhas de sene comercializadas na região de Presidente Prudente- SP, a fim de verificar se representa um potencial risco tóxico para a população usuária. As amostras analisadas permaneceram dentro das recomendações diárias para consumo tolerável dos minerais $(P, K, C a$, $\mathrm{Mg}, \mathrm{S}, \mathrm{Na}, \mathrm{B}, \mathrm{Cu}, \mathrm{Fe}$ e $\mathrm{Mn}$ ) estudados, demonstrando segurança à população usuária da folha de sene.
\end{abstract}

Palavras-chave: sene, folha, metais pesados, minerais, toxicidade.

\section{EVALUATION OF MINERAL COMPOSITION OF SENNA LEAVES BY ATOMIC ABSORPTION SPECTROMETRY}

\begin{abstract}
Some nutrients present in plants are considered essential to health by work in important metabolic pathway necessary for human life; however, it is important to note that high levels of these minerals can be dangerous and toxic to the body. The Brazilian culture has popularized the use of senna, Senna Alexandrian Miller, a plant drug used in diets for weight loss. In this sense, the objective of this study was to evaluate the concentrations of heavy metals and the contents of mineral elements by atomic spectrometry, absorption present in senna leaves sold in the region of President Prudente- SP in order to verify that is a potential risk toxic to the user population. The samples analyzed were within the tolerable daily intake recommendations for minerals $(P, K, C a$, $\mathrm{Mg}, \mathrm{S}, \mathrm{Na}, \mathrm{B}, \mathrm{Cu}, \mathrm{Fe}$ and $\mathrm{Mn}$ ) study, demonstrating the safety population that senna leaf.
\end{abstract}

Keywords: senna leaf, heavy metals, mineral toxicity.

\section{INTRODUÇÃO}

O uso de plantas medicinais é muito antigo e está intimamente relacionado à própria evolução do homem (ROMAN JUNIOR, 2003). Alguns nutrientes minerais presentes em plantas como Cobalto (Co), Cromo ( $\mathrm{Cr}$ ), Cobre (Cu), Ferro (Fe), Manganês ( $\mathrm{Mn})$, Magnésio ( $\mathrm{Mg}$ ), Selênio (Se) e Zinco (Zn) são considerados essenciais à saúde por atuarem em importantes via metabólicas, participando de atividade associada à síntese de proteína, vitamina e controle do 
metabolismo de diversas enzimas, que atuam em processos de sínteses e degradação, necessárias à vida humana (SEILER; SIGEL, 1988). Porém, é importante ressaltar que níveis elevados desses minerais podem ser perigosos e tóxicos ao organismo (SILVA, et al., 2010).

As plantas também podem acumular metais em todos os tecidos já que alguns são considerados essenciais para o seu desenvolvimento, sendo que estes podem alcançar o organismo humano, por uma das vias de exposição mais importantes: a alimentar (VEIGA et al., 2009). Existem cerca de vinte metais, considerados tóxico para os humanos, incluindo Mercúrio $(\mathrm{Hg})$, Cádmio (Cd), Paládio (Pb), Arsênio (As), Manganês (Mn), Tálio ( $\mathrm{Tl})$, Cromo (Cr), Níquel (Ni), Selênio (Se), Telúrio (Te), Antimônio (Sb), Berílio (Be), Cobalto (Co), Molibdênio (Mo), Estanho (Sn), Tungstênio (W) e Vanádio (V). Destes, os 10 primeiros são os de maior utilização industrial e, por isso mesmo, são os mais estudados no ponto de vista toxicológico (TAVARES; CARVALHO, 1992).

Os metais pesados ocorrem naturalmente, como constituintes de rochas, na água e nos seres vivos. Sendo assim, entende-se que a poluição só ocorre quando a concentração destes elementos num dado meio é bastante superior aos níveis básicos já existentes (GONÇALVES, 2000). Contudo, a grande maioria dos metais pesados aparece nos solos por contaminação resultante da atividade antropogênica (agrícola, industrial e mineira). Estes metais podem estar presentes em praguicida, fertilizantes ou resíduos de diversas origens (REICHMAN, 2002).

A cultura brasileira popularizou o consumo de sene, Senna alexandrina Miller (SIMÕES et al.,1999) uma droga vegetal utilizada em dietas para emagrecimento. Os componentes da planta, utilizados como matéria-prima, são as folhas secas, que apresentam propriedades laxativas pelo aumento do peristaltismo intestinal e da secreção de água e eletrólitos para a lúmen intestinal (EMA, 2007).

A relevância destas análises é justificada pela necessidade de conhecer o teor desses elementos para caracterizar uma possível toxicidade ao ser humano. Nesse sentido, o objetivo deste trabalho foi avaliar as concentrações de metais pesados e os teores dos elementos minerais por espectrometria de absorção atômica, presentes nas folhas de sene comercializadas na região de Presidente Prudente- SP, afim de verificar se representa um potencial risco tóxico para a população usuária.

\section{METODOLOGIA}

\section{Amostras de Chá de Sene}

No presente trabalho foram analisadas amostras de chá de Sene adquiridas em três pontos comerciais da cidade de Presidente Prudente- SP, sendo 03 (três) amostra de cada ponto, 
totalizando 9 amostras $(1,2,3,4,5,6,7,8$ e 9). Estes pontos foram escolhidos devido à credibilidade no comércio destas plantas, além de serem os únicos estabelecimentos que possuíam todas as amostras da planta escolhida. As análises foram realizadas no laboratório de farmacognosia da Faculdade de Farmácia da Universidade do Oeste Paulista (UNOESTE). Os resultados foram comparados ao modelo estatístico ANOVA com testes (LSD) $p<0,05$ para micro e macronutrientes e com valores referenciais descritos na literatura.

\section{Preparo das amostras}

Para a extração dos metais, 100g dos folíolos de sene foram trituradas e posteriormente calcinadas em mufla à temperatura de 600 C durante $12 \mathrm{~h}$, até a decomposição de toda a matéria orgânica. As cinzas obtidas foram solubilizadas em $30 \mathrm{~mL}$ de ácido clorídrico 37\%, 1:1 (v/v) e transferidas para balões volumétricos, completando-se o volume de $100 \mathrm{~mL}$ com água ultrapura. Todas as análises foram realizadas em triplicata.

\section{Determinação dos teores dos metais}

As medidas também foram realizadas em triplicata, empregando-se espectrofotometria de absorção atômica (PerkinElmer - Analyst 200). Os metais pesados analisados foram Cádmio (Cd), Chumbo $(\mathrm{Pb})$, Níquel $(\mathrm{Ni})$ e Cromo $(\mathrm{Cr})$. Foram analisados também os macronutrientes, como o Nitrogênio $(N)$, Fósforo $(P)$, Potássio $(K)$, Cálcio $(C a)$, Magnésio $(M g)$ e Enxofre $(S)$, e os micronutrientes, como Boro (B), Ferro (Fe), Manganês (Mn) Zinco (Zn) e Cobre (Cu).

\section{RESULTADOS E DISCUSSÃO}

A planta retira da solo quantidade pequenas dos elementos minerais indispensáveis ao seu crescimento, denominados macronutrientes ou micronutrientes, para completar seu ciclo vital. Entretanto, podem absorver metais pesados que são potencialmente perigosos aos homens e aos animais, por serem acumulativos no organismo.

Os resultados das concentrações de Nitrogênio total, Potássio (K), Fósforo (P), Cálcio (Ca), Magnésio (Mg), Enxofre (S), Boro (B), Cobre (Cu), Ferro (Fe) e Manganês (Mn) nas amostras da matéria seca da folha de sene estão apresentados na Tabela 1, onde se comparou as 09 (nove) amostras comercializadas em Presidente Prudente -SP, ao modelo estatístico ANOVA com teste $t$ (LSD) $p<0,05$ expressos em médias e desvio padrão, o que indicou que as mesmas apresentaram praticamente a mesma composição química, principalmente relacionado aos teores de 
micronutrientes ( $\mathrm{B}, \mathrm{Cu}, \mathrm{Fe}, \mathrm{Mn})$, e apresentou pequenas variações nos teores de macronutrientes ( $\mathrm{N}$ total, $\mathrm{K}, \mathrm{P}, \mathrm{Ca}, \mathrm{Mg}, \mathrm{S}$ ).

Essa variação de composição química está relacionada com a quantidade de nutrientes acumulados pela planta, que segundo Primavesi et al. (2002) depende da fertilidade do solo, época do plantio, além das condições climáticas.

Tabela 1. Teores dos macronutrientes ( $\mathrm{N}$ total, $\mathrm{K}, \mathrm{P}, \mathrm{Ca}, \mathrm{Mg}, \mathrm{S}$ ) e micronutrientes ( $\mathrm{B}, \mathrm{Cu}, \mathrm{Fe}, \mathrm{Mn}$ ) nas amostras da matéria seca da folha de sene em $\mathrm{g} \mathrm{kg}^{-1}$ e $\mathrm{mg} \mathrm{kg}^{-1}$ respectivamente.

\begin{tabular}{|c|c|c|c|c|c|c|c|c|c|c|c|}
\hline \multirow{3}{*}{$\begin{array}{c}\text { Amostr } \\
\text { a }\end{array}$} & \multicolumn{7}{|c|}{ Macronutrientes } & \multirow{2}{*}{\multicolumn{4}{|c|}{ Micronutriente }} \\
\hline & \multicolumn{6}{|c|}{$\mathrm{g} \mathrm{kg}^{-1}$} & \multirow{2}{*}{$\begin{array}{c}\mathrm{g} \mathrm{kg}^{-1} \\
\mathrm{Na}\end{array}$} & & & & $\mathrm{mg} \mathrm{kg}^{-1}$ \\
\hline & N TOTAL & $P$ & $\mathrm{~K}$ & $\mathrm{Ca}$ & $\mathrm{Mg}$ & $\mathrm{S}$ & & B & $\mathrm{Cu}$ & $\mathrm{Fe}$ & $\mathrm{Mn}$ \\
\hline \multirow[t]{2}{*}{1} & 22,20 & 1,56 & 8,0 & 28,3 & 3,6 & 1,8 & $* * *$ & 45,50 & 4,8 & 183,40 & 44,80 \\
\hline & & & 0 & 0 & 0 & 0 & & & 0 & & \\
\hline \multirow[t]{2}{*}{2} & 21,60 & 1,68 & 9,0 & 30,7 & 3,3 & 1,7 & $* * *$ & 38,50 & 5,1 & 242,00 & 33,60 \\
\hline & & & 0 & 0 & 0 & 0 & & & 0 & & \\
\hline \multirow[t]{2}{*}{3} & 21,70 & 1,48 & 7,6 & 28,9 & 3,5 & 2,2 & $* * *$ & 43,00 & 8,7 & 235,90 & 35,20 \\
\hline & & & 0 & 0 & 0 & 0 & & & 0 & & \\
\hline \multirow[t]{2}{*}{4} & 21,40 & 1,52 & 8.1 & 29,2 & 3,3 & 1,9 & $* * *$ & 44,00 & 6,3 & 223,40 & 34,80 \\
\hline & & & 0 & 0 & 0 & 0 & & & 0 & & \\
\hline \multirow[t]{2}{*}{5} & 22,10 & 1,49 & 7,9 & 28,4 & 3,4 & 2,0 & $* * *$ & 45,00 & 4,9 & 198,00 & 42,80 \\
\hline & & & 0 & 0 & 0 & 0 & & & 0 & & \\
\hline \multirow[t]{2}{*}{6} & 22,20 & 1,50 & 7,8 & 28,6 & 3,5 & 2,1 & $* * *$ & 44.50 & 4,8 & 190,00 & 40,00 \\
\hline & & & 0 & 0 & 0 & 0 & & & 0 & & \\
\hline \multirow[t]{2}{*}{7} & 21,80 & 1,54 & 7.4 & 29,1 & 3,6 & 1,9 & $* * *$ & 39,50 & 5,2 & 220,20 & 39,40 \\
\hline & & & 0 & 0 & 0 & 0 & & & 0 & & \\
\hline \multirow[t]{2}{*}{8} & 21,30 & 1,52 & 7,7 & 30,1 & 3,4 & 1,8 & $* * *$ & 41,00 & 6,5 & 200,40 & 35,00 \\
\hline & & & 0 & 0 & 0 & 0 & & & 0 & & \\
\hline \multirow[t]{2}{*}{9} & 21,60 & 1,60 & 8,1 & 29,7 & 3,2 & 1,8 & $* * *$ & 41,50 & 6,3 & 190,60 & 37,40 \\
\hline & & & 0 & 0 & 0 & 0 & & & 0 & & \\
\hline \multirow[t]{2}{*}{ Média } & 21,76 & 1,54 & 7,9 & 29,2 & 3,4 & 1,9 & $* * *$ & 42,50 & 5,8 & 209,3 & 38,10 \\
\hline & & & 5 & 2 & 2 & 1 & & & 4 & & \\
\hline
\end{tabular}

*** Valores não determinados

Em relação aos metais pesados, cuja a densidade é superior a $5 \mathrm{~g} / \mathrm{cm} 3$ ou que possuem número atômico maior que 20, qualquer concentração é considerada prejudicial, pois não são encontrados naturalmente e não desempenham funções nutricionais ou bioquímicas em microorganismos, plantas ou animais. No estudo foram pesquisados: Cromo ( $\mathrm{Cr}$ ), Cádmio (Cd), Níquel (Ni) e Chumbo $(\mathrm{Pb})$, porém não foram detectados teores dos metais em nenhuma das amostras.

Diversos valores disponíveis na literatura são utilizados como guia básico para interpretação do estado nutricional das plantas. Dessa forma, se o teor encontrado estiver dentro 
da faixa considerada do nutriente segundo a EMPRAPA (2009), considera-se que a cultura está bem nutrida, caso esteja abaixo ou acima, considera-se que a planta poderá apresentar problemas nutricionais relativos à deficiência ou toxidez, respectivamente. Valores máximos que podem ser ingeridos pelo organismo humano também estão disponíveis na literatura, acima destes, a Organização Mundial de Saúde $(\mathrm{WHO}, 2006)$ alerta riscos ou efeitos adversos ao organismo humano (NASCIMENTO, et al., 2013)

Para interpretação dos valores obtidos foram comparados com os valores da Recommended Dietary Allowances (RDA), esta categoria de valores deve atender às necessidades de um nutriente para 97-98\% dos indivíduos saudáveis do mesmo sexo e estágio de vida, demonstrados na Tabela 2, adaptado pela Organização Mundial de Saúde (WHO, 2006).

Tabela 2. Valores diários ( $\left.\mathrm{mg} \mathrm{dia}^{-1}\right)$ para minerais.

\section{Dose Diária Recomendada de Elemento}

\begin{tabular}{lrllllllllll}
\hline \multirow{2}{*}{ Gênero ** } & \multirow{2}{*}{ Idade } & $\mathrm{Ca}$ & $\mathrm{Cu}^{*}$ & $\mathrm{Fe} *$ & $\mathrm{Mg}$ & $\mathrm{Mn} *$ & $\mathrm{P}$ & $\mathrm{K}$ & $\mathrm{S}^{*}$ & $\mathrm{~B}^{*}$ \\
\cline { 3 - 12 } & & $\mathrm{mg}$ & $\mathrm{mg}$ & $\mathrm{mg}$ & $\mathrm{mg}$ & $\mathrm{mg}$ & $\mathrm{mg}$ & $\mathrm{mg}$ & $\mathrm{mg}$ & $\mathrm{mg}$ \\
\hline $\begin{array}{l}\text { Homem / } \\
\text { Mulher }\end{array}$ & $19-70$ anos & 1000 & 2 & 20 & 400 & 3 & 700 & 4700 & 850 & 3
\end{tabular}

FONTE: Institute of Medicine. Dietary Reference Intakes for Calcium, Phosphorous, Magnesium, Vitamin D, and Fluoride (1997); Dietary Reference Intakes for Vitamin A, Vitamin K, Arsenic, Boron, Chromium, Copper, Iodine, Iron, Manganese, Molybdenum, Nickel, Silicon, Vanadium, and Zinc (2001). Estes relatórios podem ser acessados via www.nap.edu.

** Não há alteração entre os gêneros em relação as doses diárias recomendadas para minerais

* Valores adaptado segundo a OMS (WHO,2006)

A folha de sene é consumida em forma de chás, e para avaliar os teores encontrados estão dentro dos limites seguros para o consumo humano assume-se que o consumo seja de uma xícara de chá por dia, utiliza-se $10 \mathrm{~g}$ da folha em $200 \mathrm{~mL}$ de água. Os teores ingeridos de Cálcio (Ca) das amostras seriam 283,00 mg, 307,00mg, 289,00mg, 292,00 mg, 284,00mg, 286,00mg, 291,00mg, 301,00mg, 297,00mg; de Magnésio (Mg) seria 36,00mg, 33,00mg, 35,00mg, 33,00mg, 34,00mg, 35,00mg, 36,00mg, 34,00mg e 32,00mg; de Potássio (K) 80,00 mg, 90,00mg, 76,00 mg, 81,00mg, 79,00mg, 78,00mg, 74,00mg, 77,00mg, 81,00mg e os valores de Fósforo (P) seria 15,60mg, 16,80mg 14,80 mg, 15,20mg, 14,90mg 15,00mg 15,40 mg 15,20 mg, 16,00 mg Esses valores estão abaixo do valor máximo tolerado para a ingestão diária desses minerais, segundo a RDA que recomenda 1000,00mg; 400,00mg; 4700,00mg e 700,00mg respectivamente.

Os teores das amostras de Cobre $(\mathrm{Cu})$ seriam de 0,04 mg; 0,05 mg, 0,08mg; 0,06mg, 0,04mg, 0,04mg, 0,05mg, 0,06mg e 0,06mg; Ferro (Fe) 1,83 mg; 2,42 mg e 3,35 mg 2,23mg, 1,98mg, 1,90mg, 2,20mg, 2,00 mg e 1,90; Manganês (Mn) 4,48mg, 3,36mg, 3,52mg, 3,48mg, 
4,28mg, 4,00mg, 3,94mg, 3,50mg e 3,74mg; Enxofre (S) 18,00 mg, 17,00mg, 22,00mg,19,00mg, 20,00mg, 21,00mg, 19,00mg, 18,00mg e 18,00mg e de Boro (B) seria 0,45mg, 0,38 mg, 0,43mg, 0,44mg, 0,45mg, 0,44mg, 0,39mg, 0,41mg e 0,41mg e que de acordo com OMS (WHO,2006) recomenda $2,00 \mathrm{mg}, 20,00 \mathrm{mg}, 3,00 \mathrm{mg}, 850,00 \mathrm{mg}$ e $3,00 \mathrm{mg}$ respectivamente, o que se observa que também estão abaixo do valor máximo tolerado. Diante dos resultados a utilização da folha de sene é uma fonte segura para a suplementação na alimentação humana sem risco tóxico.

\section{CONCLUSÃO}

A droga vegetal sene é bastante conhecida e acessível à população, e diante disso foram realizadas as análises com nove amostras adquiridas em diferentes pontos de comercialização: Drogaria, Farmácia de Manipulação e em Banca de Ervas, para verificar a existência de desvios no nível de metais pesados, na composição de macro e de micronutrientes, o que não se verificou na prática, indicando uma segurança toxicológica na folha de sene, em relação a composição mineral.

\section{REFERÊNCIAS}

1. EMA. Assessment Reportvon Cassia Senna L. and Cassia Anguistifolia VAHL, Folium. London: EMEA, 2007.

2. EMBRAPA- Empresa Brasileira de Pesquisa Agropecuária. Manual de análises químicas de solos, plantas e fertilizantes. Editor técnico: Fábio Cesar da Silva, $2^{\circ}$ ed. rev. ampl. Brasilia, DF: Embrapa Informação tecnológica, 2009. 627 p.

3. GONÇALVES, I.M. (2000). Fundo Geoquímico, Poluição e Comportamento do Antimónio, Cádmio, Chumbo, Crómio, Estanho e Zinco em Fluvissolos - Relatório Final de Curso de Engenharia Agronómica. Universidade Técnica de Lisboa. Lisboa.

4. Institute of Medicine. Dietary Reference Intakes for Calcium, Phosphorous, Magnesium, Vitamin D, and fluoride. Washington (DC): Nacional Academy Press; 1997.

5. Institute of Medicine. Dietary Reference Intakes for Vitamin A, Vitamin K, Arsenic, Boron, Chromium, Copper, Iodine, Iron, Manganese, Molybdenum, Nickel, Silicon, Vanadium, and Zinc. Washington (DC): Nacional Academy Press; 2001.

6. NASCIMENTO, B. L. M.; SILVA, L. D.; OLIVEIRA, J. D. de. Quantificação de Ferro e Cobre em Olerícolas oriundas de sistema orgânico e convencional. Agropecuária Científica no Semi-Árido, v.8, n.4, p 49-54 2012.

7. PRIMAVESI, O.; PRIMAVESI, A.C. \& ARMELIN, M.J.A.Qualidade mineral e degradabilidade potencial de adubos verdes conduzidos sobre Latossolos, na região tropical de São Carlos, SP, Brasil. R. Agric., 77:89-102, 2002. 
8. REICHMAN, S. M. The Responses of Plants to Metal Toxicity: A review focusing on Copper, Manganese and Zinc. Australian Minerals \& Energy Environment Foundation, 2002.

9. ROMAN JÚNIOR, W. A. Identificação de nitrocomposto e Di-Hidroflavonóis, atividades antibacteriana, antifúngica e antiviral de substâncias isoladas dos extratos liofilizados das raízes de Heteropterisaphrodisiaca O. Mach.,Malpighiaceae, Nó-de-cachorro. Araraquara: UNESP, 2003.

10. SEILER, H. G.; SIGEL, H. Handbook on toxicity of inorganic compounds. New York: Marcel Dekker, 1988.

11. SIMÕES C. M. O., Schenkel EP, Gosmann G, Mello JCP, MentzLA, Petrovick PR 1999. Farmacognosia: da planta ao medicamento. Porto Alegre:UFSC.

12. SILVA, C.S.; NUNES, P.O; MESCOUTO, CST.; MULLER, R.C.S.; PALHETA,D.C.; FERNANDES,K.G. Avaliação do uso das cascas do fruto e das folhas de Caesalpinia férrea Martius como suplemento nutricional de $\mathrm{Fe}, \mathrm{Mn}$ e Zn. Ciênc.Tecnol.Aliment, v.30,n.3, p.751-754,2010. http://dx.doi.org/10.1590/S0101-20612010000300028

13. TAVARES TM, Carvalho FM 1992. Avaliação da exposição de populações humanas a metais pesados no ambiente: exemplos do Recôncavo Bahiano. Quim Nova 15: 147-153.

14. VEIGA, A.; LOPES, A.; CARRILHO, E.; SILVA, L.; DIAS, M. B.; SEABRA, M. J.; BORGES, M.; FERNANDES, P.; NUNES, S. Perfil de risco dos principais alimentos consumidos em Portugal. Autoridade de Segurança Alimentar e Económica. Direção de Avaliação e Comunicação dos Riscos , 2009

15. WHO-WORLD HEALTH ORGANIZATION. Guidelines on food fortification with micronutrients. WHO/FAO. 2006. 376 p. Disponível em: http://www.who.int/nutrition/ 